\title{
Frontières
}

\section{Musique et funérailles chez les Dàgàrà-Lòbr du Burkina Faso}

\section{David Vaulay}

Volume 20, numéro 2, printemps 2008

Les musiques et la mort

URI : https://id.erudit.org/iderudit/018334ar

DOI : https://doi.org/10.7202/018334ar

Aller au sommaire du numéro

Éditeur(s)

Université du Québec à Montréal

ISSN

1180-3479 (imprimé)

1916-0976 (numérique)

Découvrir la revue

Citer cet article

Vaulay, D. (2008). Musique et funérailles chez les Dàgàrà-Lòbr du Burkina Faso. Frontières, 20(2), 49-54. https://doi.org/10.7202/018334ar

\section{Résumé de l'article}

Cet article a pour but de montrer la place et le rôle, en particulier cathartique, de la musique dans les cérémonies funéraires des Dàgàrà-Lòbr du Burkina Faso. Il présente les ensembles instrumentaux qui y sont joués et dont les instruments les plus importants sont les xylophones. L'auteur y décrit les principales étapes des funérailles, du décès jusqu'à l'inhumation. Dans cette description est mise en relief l'articulation des différentes phases rituelles avec la musique des deux ensembles de xylophones dont l'un, ensemble lóbrí, intervient pendant les phases liminaires et l'autre, ensemble dègàar, durant la cérémonie proprement dite et sert de support à des louanges, lamentations et autres paroles de consolation prononcées par un groupe d'hommes appelé làng-kóndbè ou cantateurs. 


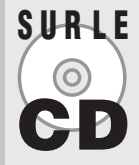

Làngnin des cantateurs lors de funérailles au Burkina Faso, en février 2006. Enregistrement: David Vaulay.

Durée: $9 \min 14 \mathrm{~s}$

Résumé

Cet article a pour but de montrer la place et le rôle, en particulier cathartique, de la musique dans les cérémonies funéraires des Dàgàrà-Lòbr du Burkina Faso. II présente les ensembles instrumentaux qui y sont joués et dont les instruments les plus importants sont les xylophones. L'auteur y décrit les principales étapes des funérailles, du décès jusqu'à l'inhumation. Dans cette description est mise en relief I'articulation des différentes phases rituelles avec la musique des deux ensembles de xylophones dont l'un, ensemble lóbrí, intervient pendant les phases liminaires et l'autre, ensemble dżgàar, durant la cérémonie proprement dite et sert de support à des louanges, lamentations et autres paroles de consolation prononcées par un groupe d'hommes appelé làngkóndbè ou cantateurs.

Mots clés: Dàgàrà - xylophone funérailles - cantateurs.

\section{Abstract}

The purpose of this article is to show the place and the role, cathartic in particular, of music in the mortuary ceremonies among the Dàgàrà-Lòbr from Burkina Faso. It presents the instrumental ensembles which are played there and whose most important instruments are the xylophones. The author describes the main stages of the funeral, from death until the end of the ceremony. In this description is highlighted the articulation of the various ritual phases with the music of the two sets of xylophones, namely the lóbrí ensemble which intervene during the preliminary phases and the dìgàar ensemble which is played during the ceremony itself and is used as a support to praises, lamentations and other words of comfort pronounced by a group of men called làng-kóndbغ̀ or cantateurs.

Keywords: Dàgàrà - xylophone funeral - cantateurs.

\section{Musique et funérailles chez les Dàgàrà-Lòbr du Burkina Faso}

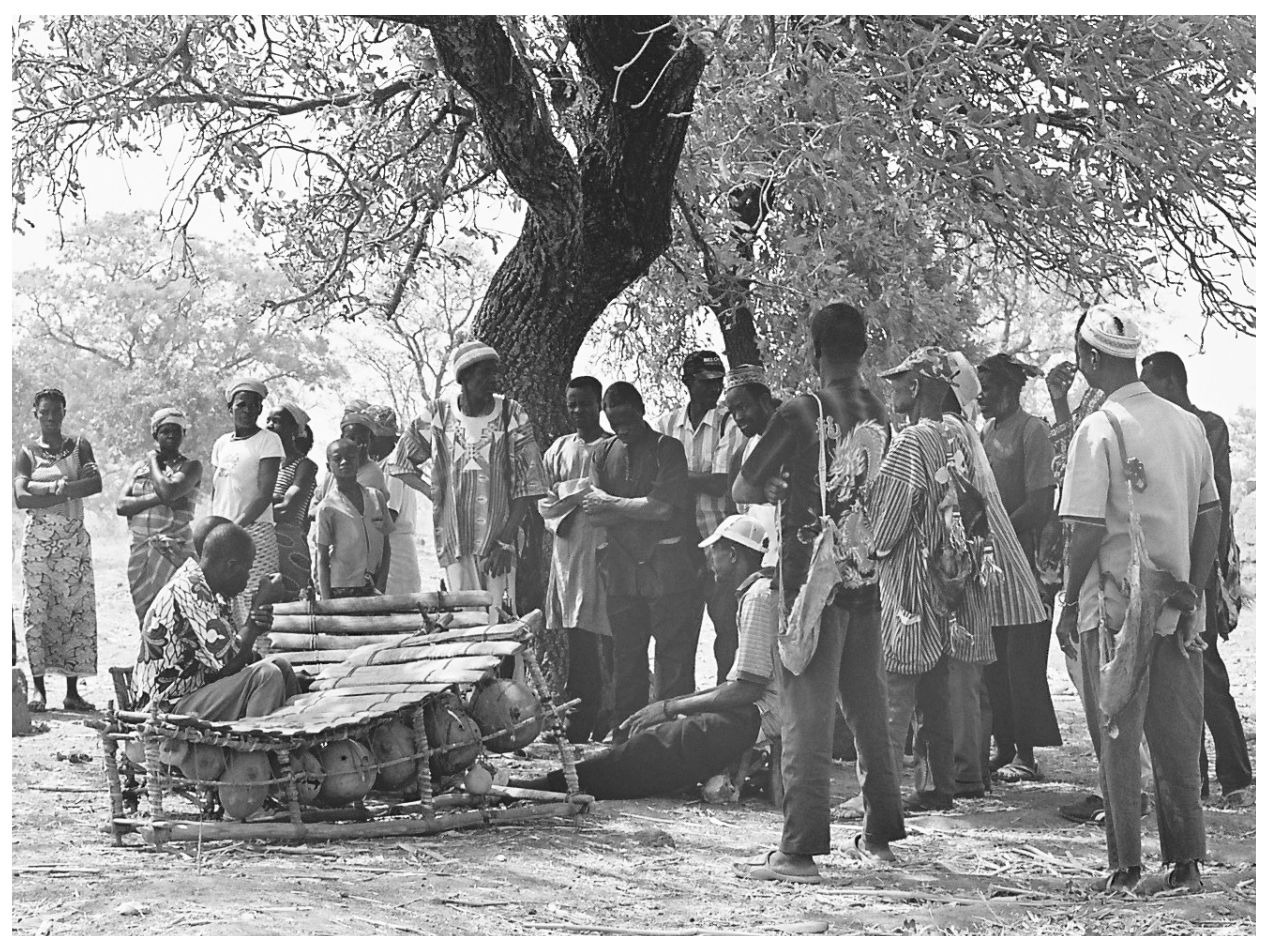

L'ensemble dègàar et les cantateurs.

\section{David Vaulay, \\ étudiant en Master 2 d'ethnomusicologie, Université de Paris X Nanterre.}

Les Dàgàrà vivent au sud-ouest du Burkina Faso et au nord-ouest du Ghana. Les recherches ethnomusicologiques les concernant sont peu nombreuses et ont pour l'essentiel été menées auprès des Dàgàrà du Ghana par des chercheurs anglophones. D'un point de vue strictement anthropologique, la contribution la plus importante demeure celle de Jack Goody, en particulier Death, Pro- perty and the Ancestors (Goody, 1962), qui propose une étude approfondie de l'organisation sociale dàgàrà (qu'il nomme «LoDagaa») à travers l'analyse de leurs coutumes funéraires.

Le présent article s'appuie sur une ethnographie menée chez les Dàgàrà-Lòbr, sousgroupe Dàgàrà, au Burkina Faso autour de la petite ville de Dissin, près de la frontière ghanéenne. Il constitue un premier jalon vers une étude ethnomusicologique des funérailles dàgàrà, des instruments et des répertoires qui y sont joués. Après la présentation 
des instruments de musique et de ce que les Dàgàrà appellent les cantateurs, nous présenterons les grandes étapes de la cérémonie en mettant l'accent sur la place que tient la musique ainsi qu'une première analyse de son implication dans le rite funéraire ${ }^{1}$.

\section{LES INSTRUMENTS}

Le xylophone ${ }^{2}$ est l'instrument le plus important chez les Dàgàrà où il est de deux types: sur fosse (principalement joué par les enfants pour l'apprentissage de l'instrument) et sur cadre. Chez les Lòbr, le xylophone sur cadre à résonateurs multiples en calebasse et à clavier linéaire (c.-à-d. dont l'ordre des hauteurs est successif) se décline en deux exemplaires: le lóbrí et le dègàar.

Le xylophone lóbrí comporte un clavier de 14 lames, accordées selon une échelle pentatonique hémitonique ${ }^{3}$. Il est toujours joué par paire et est accompagné, lors des funérailles uniquement, d'un membranophone cylindrique appelé gàngàar.

Le xylophone ḋ̀gàar comporte un clavier de 17 (parfois 18) lames accordées selon une échelle pentatonique anhémitonique $^{4}$. Il est accompagné du júl kpàwrè et du kúor. Le jíl kpàwrè fait partie des idiophones par percussion. Il consiste en une à trois des lames les plus graves du xylophone, percutées avec une paire de baguettes de bois. Quant au kv́or, il s'agit d'un membranophone sur calebasse de la famille des timbales.

Ces deux ensembles, que je nommerai «ensemble lóbrí » et "ensemble dègàar », interviennent à des moments différents des funérailles.

\section{LES LÀNG-KÓNDBÈ OU CANTATEURS}

Les làng-kóndbè forment un groupe d'hommes qui se rassemblent près de l'ensemble dègàar lorsque celui-ci intervient. Selon Constantin Gbãanè Dabire, ce moment constitue «un rite de lamentations et de pleurs accompagnés de danses» (Gbãanغ̀ Dabire, 1983, p. 272). En effet, leurs interventions, làngnìn ${ }^{5}$ sont faites de lamentations, de louanges, voire de critiques ainsi que de récits rappelant des épisodes de la vie du défunt auxquels se mêlent proverbes et devises claniques. Le terme làng-kóndbè (làng-kónné au singulier $)^{6}$ est traduit par les Dàgàrà lorsqu'ils s'expriment en français par le mot cantateurs ${ }^{7}$.

$\mathrm{Du}$ groupe des cantateurs se détachent un certain nombre de "solistes», dont généralement deux principaux, qui interviennent alternativement selon leur inspiration, en se répondant parfois. À propos du discours des làng-kóndbè, Jack Goody précise : "the funeral chant (long), though it always includes standardized phrases of a proverbial kind, is largely impromptu» (1962, p. 100). Bien que ce dernier parle de funeral chant, les Dàgàrà ne considèrent ces louanges et lamentations ni comme de la musique ni comme du chant ${ }^{8}$. De fait, les interventions des solistes sont beaucoup plus proches du parlé que du chanté, bien que de courtes formules mélodiques viennent parfois se placer en début et en fin de phrase. Le reste des làng-kóndbè forme un chœur qui ponctue le discours des solistes par des phrases mélodiques stéréotypées de courbe descendante sur des syllabes sans signification. La forme peut donc être considérée comme responsoriale ${ }^{9}$.

\section{LES FUNÉRAILLES}

Leurs différentes phases seront présentées ici à partir du constat du décès jusqu'à l'inhumation, étant entendu que certaines de ces phases sont susceptibles de se chevaucher et que leur ordre de succession est plus souple qu'il n'y paraît.

La cérémonie type présentée est pratiquée pour le décès d'un adulte, c'est-àdire de tout homme circoncis ou de toute femme ayant eu ses premières menstruations. Selon l'âge et l'importance du défunt, les funérailles dureront deux, trois voire quatre jours dans le cas de personnes très âgées.

\section{L'ANNONCE DU DÉCÈS}

Le décès est annoncé de trois façons différentes selon qu'il s'agit de prévenir la famille proche, les personnes vivant alentour ou encore celles qui sont plus éloignées.

\section{LES PLEURS, LES CRIS ET LA CENDRE}

Les pleurs sont soumis à des règles. Excepté pour le cas du décès d'un enfant de quelques semaines, une femme ne doit jamais pleurer en public avant que le plus vieil homme, c'est-à-dire le chef de famille n'ait été prévenu et n'ait luimême pleuré. Cet homme peut être le père, le plus vieux des frères ou même le plus vieux des enfants du défunt, s'il est le plus âgé de la famille. Dans ce dernier cas, il faut toutefois qu'il s'agisse d'un dèblé (de dèb, homme, et lé, petit), c'est-à-dire qu'il ait un frère ou une sœur puîné. Si une femme meurt, c'est son mari qui doit pleurer le premier. Si le mari est en voyage, c'est le père, le plus vieux des frères du mari ou encore le plus vieux de ses fils.

La tradition veut que, la main enduite ou remplie de cendres, l'on frappe la nuque de l'homme qui doit pleurer le premier. Au moment où l'on fait ce geste le décès lui est annoncé. Il s'agit d'un geste de consola- tion, la cendre ayant, pour les Dàgàrà, des propriétés apaisantes et consolatrices.

Ayant appris la nouvelle, l'homme lance alors un cri stéréotypé: «Sàan wó $i$ » ( Oh mon Père!») ou «Màn wó ì! « («Oh ma Mère!»). Les autres personnes peuvent alors commencer à pleurer également.

\section{LE JEU DE L'ENSEMBLE LÓBRÍ}

L'ensemble lóbrí est le premier à être joué avant même que l'on envoie des messagers pour annoncer les funérailles. Il ne semble toutefois pas y avoir de prescription pour ce qui est du moment où l'on commence à jouer, si ce n'est que l'homme qui doit pleurer en premier ne doit en aucun cas entendre le son des xylophones avant d'avoir été prévenu.

L'ensemble lóbrí, et tout particulièrement le tambour gàngàar, annonce qu'un décès est survenu. En effet, le son de celui-ci est particulièrement puissant et porte à plusieurs kilomètres. Or le gàngàar annonce non seulement le décès mais aussi le sexe du défunt à l'aide de deux courtes cellules rythmiques ayant pour noms dèblú ( "mâle») pour le décès d'un homme et pc̀glv́ («féminité») pour une femme.

Le xylophone lóbrí présente une particularité liée aux lames 5 et $10^{10}$, appelées gàng-bèrà ("dépasser laisser»). Ces lames ne sont jouées que lors des funérailles, la nuit, à un moment bien défini et dans un but précis, comme nous le verrons plus loin, et encore ne le sont-elles pas systématiquement. En dehors de ces moments, seules douze des quatorze lames sont jouées. Musicalement, cela implique que l'essentiel de la musique jouée sur xylophone lóbrí est tétratonique, c'est-à-dire utilisant une échelle de quatre sons.

Quatre répertoires sont joués par l'ensemble lóbrí lors des funérailles : dèblv́ avec et sans les lames gàng-bèrà et pc̀glpc̀ avec et sans ces mêmes lames.

\section{LES MESSAGERS}

Des hommes du village, le plus souvent des jeunes, sont envoyés pour annoncer le décès dans les environs à la famille, aux amis et autres connaissances.

Lorsque les proches ont été prévenus, le corps est apprêté pour la cérémonie par des femmes (défunte) ou des hommes, parfois les fossoyeurs (défunt). Selon J. Goody, la toilette du défunt doit être faite par des épouses et sœurs classificatoires (c'est-à-dire par des femmes n'étant pas trop proches du mort). L'auteur précise également:

[I]t is prescribed that these women should have passed the menopause, that they should, to use the LoDagaa phrase, have "turned to men" (lieba daba). They can no longer perform the 
main task of women, the bearing of children, and are, as it were, asexual. This attribute permits them to carry out intimate physical acts on members of both sexes, an attribute that in Western European society is ideally associated with the doctor (Goody, 1962, p. 56).

Ceci reste à vérifier dans le cas des Dàgàrà-Lòbr.

\section{LA PRÉPARATION DU CORPS}

Le défunt est lavé avec de l'eau et du savon. Son crâne est rasé et son visage enduit de talc afin d'éloigner les mouches et de diminuer les problèmes d'odeur en cas de début de décomposition du cadavre avant l'enterrement. Son bassin est enveloppé d'une cotonnade blanche, longue et étroite bande dont les extrémités sont coupées en trois parties pour un homme et en quatre pour une femme. Cette symbolique est répandue dans toute l'Afrique de l'Ouest, parfois de façon inverse (homme: 4, femme: 3). L'explication qui m'a été donnée est que l'homme répète ses actes trois fois et la femme quatre fois. Germaine Dieterlen, dans Essai sur la religion bambara, avait déjà relevé cette explication mais liée, dans son cas, à une symbolique sexuelle assez courante, le chiffre 3 renvoyant à la verge et aux testicules, le chiffre 4 aux quatre lèvres de la femme (Dieterlen, 1950). Aucune référence à une symbolique sexuelle ne m'a toutefois été rapportée sur le terrain.

Enfin, les fossoyeurs plient les membres du cadavre pour pouvoir l'habiller du costume traditionnel dàgàrà .

\section{L'EXPOSITION DU DÉFUNT DEVANT SA MAISON}

Le défunt apprêté est alors installé par les fossoyeurs dans un siège adossé à un mur près de l'entrée principale, à l'extérieur de sa maison qui est un espace public. Sous ce siège, une natte en épis de mil permet que les pieds du défunt ne touchent pas le sol, ce qui serait un manque de respect. En effet, il fut un temps où l'absence de natte désignait l'esclave ou l'inconnu. D'autre part, le corps ne doit pas toucher la terre qui lui a donné la vie et qui ne la reprendra qu'au moment de l'inhumation. Le défunt est installé avec les attributs de son sexe, symboles de sa réalisation en tant qu'homme ou femme. Les hommes portent un arc sur les genoux et, en bandoulière, un carquois contenant quelques pailles servant à fabriquer les flèches mais sans pointe et trois vraies flèches (3, chiffre lié à l'homme) dont il pourra faire usage s'il croise le fantôme de l'un de ses ennemis lors de son voyage vers le

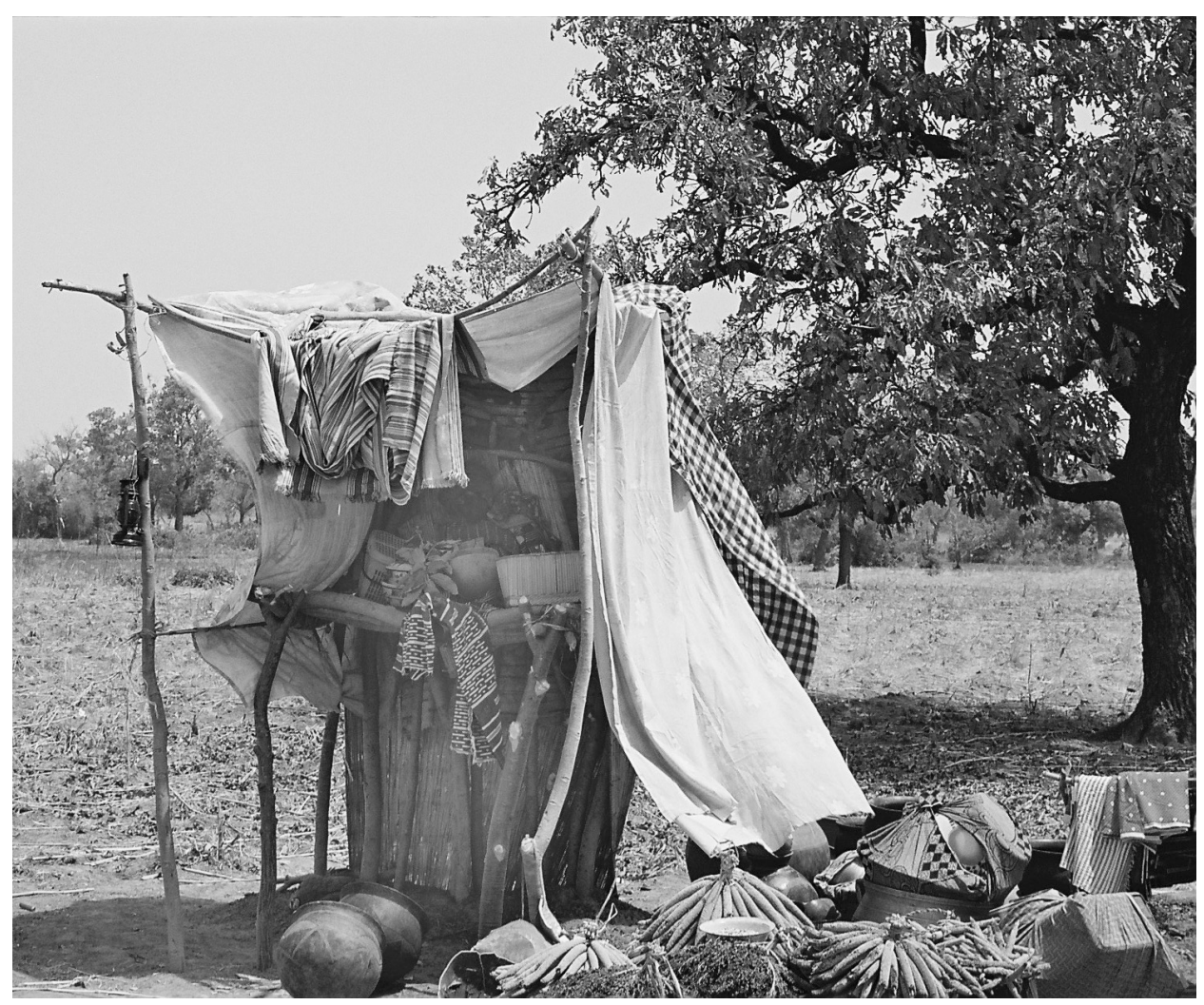

Le catafalque entouré des symboles de la réussite du défunt.

Pays des Ancêtres. Les femmes portent une calebasse sur les genoux dans laquelle sont placées leurs mains.

Si la cérémonie n'a pas à proprement parler commencé, on entre d'ores et déjà dans la phase publique des funérailles. Lorsqu'une personne arrive aux funérailles, elle va saluer le défunt et la famille endeuillée. Cette salutation se fait par des «va-et-vient» devant le défunt: on passe devant le corps, on le dépasse et marque alors une pause, on fait ensuite de même dans l'autre sens et enfin on retourne une dernière fois sur ses pas pour venir s'arrêter et se recueillir devant le corps. Enfin on s'approche du défunt pour jeter de l'argent à ses pieds, et l'on va jeter également un peu de monnaie aux musiciens (deux xylophones lóbrí et un tambour gàngàar) installés non loin de là. D'après mes observations lors de diverses funérailles, j'ai pu remarquer qu'autour de la chaise mortuaire se tiennent généralement les femmes dans les pleurs et les cris, tandis que les hommes, moins expansifs, forment un autre groupe légèrement en retrait face au mort.

Peu à peu, le défunt se trouve entouré de paniers remplis de mil apportés par chaque homme de la famille possédant un grenier. Le défunt transmettra ce mil aux ancêtres comme témoignage de la vitalité de leurs descendants. Il sera partagé entre les fossoyeurs à la fin des funérailles. Pour une défunte, les femmes de la famille apporteront canaris, paniers ou encore nattes.
La durée de cette phase d'ouverture de la cérémonie mortuaire dépend du moment du décès: s'il a eu lieu avant midi, elle durera jusqu'à 15 heures environ, mais s'il a eu lieu à midi ou après, elle se prolongera jusqu'au lendemain matin. Le défunt sera alors installé sur un catafalque.

\section{L'EXPOSITION DU DÉFUNT SUR LE CATAFALQUE}

Le catafalque est une construction en bois sur laquelle est «exposé» le corps. Construit non loin de la concession, à un endroit où l'assistance peut s'installer à l'ombre sans être toutefois trop proche du défunt, le catafalque est orienté vers l'est pour les hommes (qui se lèvent tôt pour aller aux champs), et vers l'ouest pour les femmes (qui savent, lorsque le soleil commence à descendre, qu'il faut préparer le repas).

Lorsque les fossoyeurs ont achevé la construction, et selon l'heure du décès, ils rentrent le corps dans la maison où ils lui plient à nouveau les membres afin de pouvoir le disposer plus aisément sur le catafalque. Lorsqu'ils ressortent pour aller l'installer, toute l'assistance se lève, y compris les musiciens qui arrêtent alors de jouer, et se tournent vers le défunt en signe de respect.

Le corps est disposé sur la plate-forme du catafalque, dos appuyé contre le fond. Autour du catafalque sont exposés les symboles de la réussite du défunt. Pour un 
homme : du mil provenant de ses champs, ses malles, fusils, trophées de chasse, etc.; pour une femme: du mil également, ses ustensiles de cuisine ou encore ses tissus. On peut également voir un certain nombre d'animaux attachés au catafalque ou non loin : un des bœufs du défunt portant à son cou le manche d'une houe et qui témoigne de sa réussite agricole; un bœuf apporté par les membres du matriclan qui sera rituellement tué et partagé entre les membres des matriclans; une chèvre apportée par les frères propres de la défunte et qui témoigne du fait que celle-ci rapportait de l'argent à la famille; ou encore une chèvre apportée par les enfants de la défunte symbolisant son rôle de mère (qui récoltait des feuilles pour nourrir ses enfants), etc.

Dans La mort africaine, Louis-Vincent Thomas souligne que «le mort est évidemment le personnage principal autour duquel s'articulent tous les rites funéraires » et qu'«il préside les rites, entouré de ce qui fut le symbole de son existence [...] » (Thomas, 1982, p. 150). C'est bien de cette façon que cela se passe lors des funérailles dàgàrà où le défunt préside ses propres funérailles depuis son "trône de bois" (Gbãanc̀ Dabire, 1983, p. 270).

\section{LA CÉRÉMONIE FUNÉRAIRE}

L'assistance, qui a suivi les fossoyeurs et le défunt, s'est regroupée par village et s'est installée à l'ombre des arbres. Les musiciens s'installent, également à l'ombre, à quelques dizaines de mètres en face du catafalque et continuent à jouer les xylophones lóbrí accompagnés du tambour gàngàar.

\section{LE JEU DE L'ENSEMBLE DغेGÀAR}

Lorsque la famille estime que l'assemblée est assez nombreuse, elle décide de changer d'orchestre, c'est-à-dire de passer à l'ensemble dègàar et au làngnìn ${ }^{5}$ des cantateurs. C'est alors le véritable commencement de la cérémonie funéraire.

Selon le sexe du défunt et, dans le cas d'une femme, sa condition sociale, l'ordre et la structure des interventions des musiciens et des cantateurs diffère. Pour le décès d'une femme non mariée ou d'un homme, ceux qui interviennent les premiers font partie de la famille paternelle, c'est-à-dire du même patriclan que le défunt. Dans le cas d'une femme mariée et dotée, c'est le patriclan du mari qui commence suivi de celui du père (ou l'inverse si la femme n'était pas encore dotée). Dans ces trois cas, tandis que les cantateurs lancent leurs louanges, les musiciens jouent 3 pièces du répertoire dègàar pour le décès d'un homme, 4 pour une femme, en marquant une pause entre chaque pièce.
Puis ils jouent «un bèlàngnèn », c'est-àdire un nombre non défini de pièces du répertoire bèlàngnèn enchaînées les unes aux autres ${ }^{11}$.

Lorsque deux dègàar ont été joués, tout le groupe se déplace en dehors de l'aire du catafalque pour aller s'installer à l'endroit où était joué l'ensemble lóbrí quelques minutes plus tôt. Le ou les dègàar restants sont alors joués ainsi que le bèlàngnèn. La place est ensuite cédée aux cantateurs et aux musiciens du matriclan du défunt qui jouent deux dìgàar et un bèlàngnèn. Puis se succéderont les membres des villages présents aux funérailles du plus proche au plus lointain. Les musiciens de ces villages interprètent eux aussi deux dègàar et un bèlàngnèn, avec leurs cantateurs. Chaque intervention dure généralement environ 45 minutes et est accompagnée de danses.

Le moment où l'on joue le répertoire bèlàngnèn est particulièrement intense. En effet, en plus des danseurs, la foule se presse vers les musiciens et les cantateurs. On donne alors de la monnaie aux musiciens et à un parent du défunt qui se trouve parmi les cantateurs. Il est alors de coutume de rester près d'eux jusqu'à la fin du bèlàngnèn.

C'est le parent qui se trouve parmi les cantateurs qui décide du moment où musiciens et cantateurs s'arrêtent. Pour ce faire, il partage l'argent qu'il a reçu en deux et en jette une moitié au sol. L'argent qui est au sol revient aux musiciens et l'autre partie aux cantateurs. Alors chacun rejoint sa place à l'ombre des arbres et un autre groupe de musiciens et de cantateurs prend place.

\section{LA VEILLÉE NOCTURNE}

Lorsque la nuit tombe, les fossoyeurs replacent le mort dans la chaise contre le mur de sa maison. L'ensemble dègàar est alors à nouveau joué (deux dègàar et un bèlàngnèn) par des membres du patriclan du père ou du mari selon les cas exposés plus haut. Après quoi l'ensemble lóbrí sera joué durant toute la nuit.

À minuit, il peut arriver que l'on joue des pièces des répertoires dèblv́ ou pc̀glv́ avec les lames gàng-bèrà. Ces pièces sont jouées afin de convier les ancêtres aux funérailles. Ce moment est le seul où toutes les lames des xylophones lóbrí sont jouées et où leur musique est pentatonique. Même lors des funérailles, ces lames restent cependant rarement jouées pour deux raisons: d'une part, peu de musiciens savent les jouer et, surtout, faire venir les ancêtres aux funérailles n'est pas sans danger. En effet, si ceux-ci venaient à constater une dégradation de la situation de la famille, par exemple, des représailles pourraient s'en suivre.

\section{LA CLÔTURE DES FUNÉRAILLES}

\section{ZÁNNV̀, RITE DE COMMÉMORATION} ET DE SÉPARATION

La dernière journée des funérailles est marquée par un rite de commémoration ${ }^{12}$ appelé zánnv́ (rêve). Durant ces commémorations, les compagnons de travail, de chasse, de loisirs ou encore d'initiation du défunt se regroupent pour reproduire les activités et gestes qu'ils avaient coutume de faire avec leur ami. Parmi les scènes auxquelles j'ai pu assister lors de diverses funérailles figurent celles de femmes plantant du riz ou encore une véritable manifestation de femmes brandissant des pancartes et criant des slogans et dont on m'a expliqué qu'elles avaient coutume de faire des «réunions de femmes» avec la défunte. Zánnv́ est, à ma connaissance, le seul moment où l'on peut entendre des musiques qui ne sont pas liées aux funérailles comme la flûte vùlò appelant les chasseurs à se rassembler ou encore des chants du répertoire núrú chanté par les femmes habituellement lors de fêtes marquant la fin des récoltes.

Le nom donné à ce rite, zánnú « rêve», ou, selon C. Gbãanc̀ Dabire, «zánnv́-iru» (Gbãanغ̀ Dabire, 1983, p. 275), littéralement «rêve ôter », vient de ce que ces commémorations sont indispensables pour que le défunt ne revienne pas hanter les rêves de ses anciens compagnons.

Plus encore, comme le souligne LouisVincent Thomas, «en tant qu'elles [les funérailles] doivent consacrer la coupure introduite par la mort, elles coïncident avec la mise en place d'un rituel de séparation qui trouve son prolongement dans le temps avec la période de deuil » (Thomas, 1982, p. 149). Zánnv́ constitue bien un rite de séparation. Il s'agit non seulement «de réjouir le défunt pour une dernière fois au spectacle de sa vie sur terre» mais aussi de lui signifier son congé qui sera encore affirmé par l'inhumation, "rupture sans appel des liens d'ordre terrestre avec le défunt»(Gbãanè Dabire, 1983, p. 276).

Zánnv́ consacre donc la séparation entre le défunt, qui va rejoindre le Pays des Ancêtres, et la communauté qui va devoir procéder à certains réajustements.

\section{L'INHUMATION}

Le moment de l'inhumation est choisi par la famille du défunt. Elle a généralement lieu en fin de journée avant le coucher du soleil ${ }^{13}$. Les fossoyeurs, qui, durant la cérémonie, ont creusé la tombe, s'enduisent les bras de terre mouillée afin d'éviter tout contact direct avec le cadavre. Le moment où ils prennent possession du défunt pour aller l'inhumer est un moment intense et douloureux. C'est celui des derniers adieux. 
L'endroit où est creusée la tombe est désigné par le chef de terre ou par la famille ${ }^{14}$, les vieillards étant inhumés dans la cour de leur maison. Entre autres gestes rituels pratiqués par les fossoyeurs avant de creuser une tombe, ceux-ci posent une calebasse renversée au sol. Sur son pourtour est versée de la cendre. Le cercle ainsi tracé détermine l'entrée de la tombe sous laquelle cette dernière est creusée par tous les fossoyeurs à tour de rôle.

L'inhumation a lieu à l'abri des regards. Pour cela, les fossoyeurs cachent la scène en formant un écran à l'aide de tissus qu'ils tiennent tout autour de la tombe.

Le défunt est disposé sur le flanc, face vers l'est pour les hommes, vers l'ouest pour les femmes. Les poches de son habit sont déchirées. Nous venons en effet au monde sans argent et devons en repartir de même. C. Gbãanè Dabire explique d'autre part «qu'aucun des cadeaux offerts au défunt ne l'accompagne dans la tombe [...]. Il est parti dans l'au-delà où il a bel et bien emporté la "substance" invisible des présents offerts : à la clôture de la veillée, on détruira les "enveloppes" visibles (fòfór) des objets, qui ne sont plus que des "apparences" de choses sans consistance [...]». Toutefois, dans la majorité des cas, les présents ne sont pas détruits mais récu- pérés et ce, semble-t-il, pour des raisons essentiellement économiques.

L'ouverture scellée avec une pierre ou un canari percé, dans le cas d'un défunt n'ayant plus de frère ou de sœur propre, la tombe est enfin recouverte avec la terre qui en a été extraite.

Les fossoyeurs vont alors détruire le catafalque, moment durant lequel les musiciens repassent l'ensemble lóbrí. Puis les musiciens et les cantateurs de la famille du père ou du mari jouent l'ensemble dègàar (trois ou quatre dègàar selon le sexe du défunt et un bèlàngnèn) et "chantent» une dernière fois leurs louanges et lamentations. Enfin pendant le bèlàngnèn est marquée la fin des funérailles par un geste des bras de haut en bas de la part d'un «parent à plaisanterie».

Le plus souvent l'assistance restera encore un long moment sur le lieu des funérailles qui se transforme alors fréquemment en marché, lieu où les Dàgàrà ont l'habitude de se retrouver pour discuter autour d'une calebasse de bière de mil.

\section{LE RÔLE DE LA MUSIQUE \\ DANS LES FUNÉRAILLES: PREMIERS ÉLÉMENTS D'ANALYSE}

Le rôle premier de l'ensemble lóbrí, et particulièrement du membranophone cylindrique gàngàar, semble bien être signalétique: à son audition, on sait qu'un décès vient de survenir, où il a eu lieu et quel est le sexe de la personne décédée. D'autre part, cet ensemble est joué pendant la phase liminaire de la cérémonie qui va de l'annonce du décès au véritable déclenchement de la cérémonie quand, le défunt étant sur le catafalque, les cantateurs commencent le làngnìn accompagné par l'ensemble dègàar. Elle est à la fois un moment de préparation (préparation du défunt pour la cérémonie et préparation de la cérémonie elle-même: annonce de la nouvelle, construction du catafalque, etc.) et un moment d'attente, principalement de l'arrivée de l'assistance sur le lieu de la cérémonie. La nuit, durant laquelle sont également joués les xylophones lóbrí, ressemble également à une phase d'attente: le défunt, s'il reste le point focal, ne préside plus comme du haut du catafalque, il n'y a plus de cantateur et dans l'ensemble, l'intensité est réduite, beaucoup de gens finissant par aller dormir. De même, les moments où le défunt est sur le catafalque, où il n'y a pas de cantateurs disponibles et où l'on repasse du dègàar aux lóbrí semble bien être également un moment d'attente: attente que des cantateurs viennent « chanter» leurs louanges et lamentations.

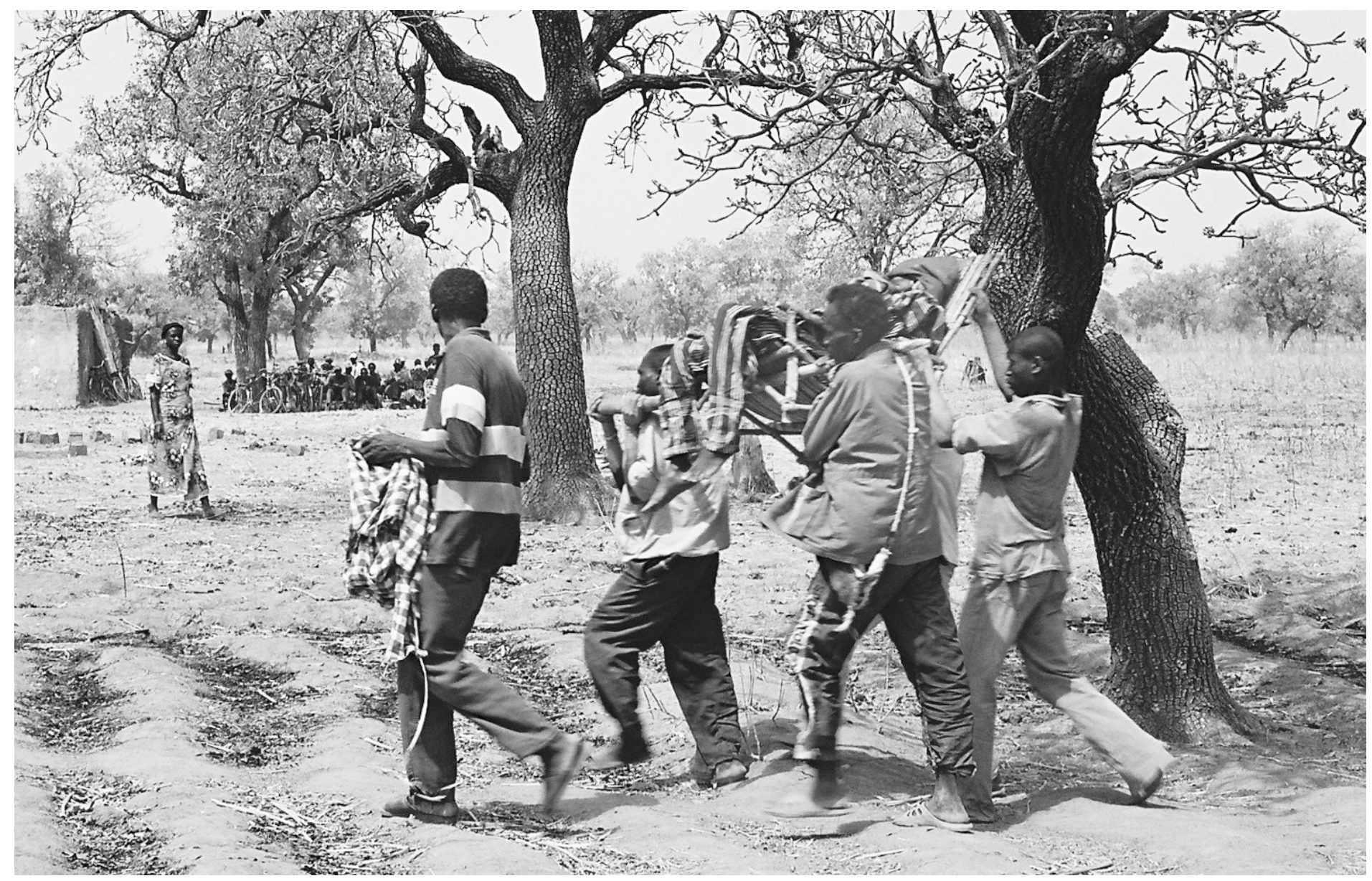

Les fossoyeurs transportant le défunt pour l'inhumation. 
Enfin, et cela va dans le sens d'une compréhension des funérailles comme catharsis, il y a, selon le point de vue même des Dàgàrà, une différence majeure entre les lóbrí et le dègàar: les premiers font pleurer les gens, alors que ce sont les cantateurs qui font pleurer lorsque l'on joue le xylophone dègàar, et non le dègàar lui-même. On trouve dans Jalons pour une ethnomusicologie dagara une comparaison assez parlante: d'une part, "la musique du "lobri" [...] est une musique à tons mineurs. C'est une musique triste et mélancolique» (Somé et Bekuone, 1976, p. 39), d'autre part, «le balafon dègàar évolue [...] dans une gamme majeure» (p. 59), il est «plus solennel, sacré et majestueux» (p. 41). Si d'un point de vue théorique, la comparaison entre les échelles des xylophones dàgàrà et les échelles majeure et mineure de la musique classique occidentale n'est pas possible telle quelle, en revanche le parallèle avec le mineur en tant qu'il est souvent considéré comme mélancolique, triste et sombre et le majeur qui serait plus lumineux et majestueux fonctionne très bien et rend tout à fait compte de la différence entre les deux xylophones telle qu'elle m'a été expliquée par mes informateurs. C. Gbãanè Dabire ajoute quant à lui que les cantateurs, qui sont « experts dans l'art d'exciter les sentiments, se chargent d'attendrir les cœurs les plus indifférents $[\ldots]$ » et que «le but du gyil-gub [moment des funérailles où se font entendre l'ensemble dègàar et les cantateurs accompagnés de pleurs et de danses] est de manifester la douleur et le désarroi de la communauté face au départ d'un de ses membres » (Gbãanc̀ Dabire, 1983, p. 273). Aussi est-il permis de penser que le fait de repasser à l'ensemble lóbrí lorsqu'il n'y a pas de cantateurs répond à ce besoin cathartique d'extérioriser «la douleur et le désarroi», ce que le xylophone dغ̀gàar seul ne permettrait pas.

Quant aux làng-kóndbè, ils n'interviennent jamais sans l'accompagnement de l'ensemble dègàar qui sert de support au làngnìn. Les cantateurs sont investis d'une dimension sociale particulière. En effet, comme nous l'avons vu plus haut, l'ordre d'intervention des différents groupes est lié à la plus ou moins grande perte que constitue le décès pour chacun et réaffirme une hiérarchie dans l'appartenance de chaque individu aux différents groupes sociaux en présence. D'autre part, les louanges et lamentations des cantateurs renvoient à l'histoire personnelle du défunt. Enfin, les musiciens et cantateurs jouant et «chantant» pour le défunt mais aussi pour les familles, ils contribuent à maintenir ou renforcer les liens sociaux existant entre les différents groupes sociaux en présence, patriclan et matriclan, mais aussi entre les villages.

Comme l'avait déjà montré Jack Goody (1962), les funérailles sont le point de convergence de toutes les dimensions fondamentales de la culture, réaffirmées lors de chaque cérémonie: places respectives de l'homme et de la femme dans la société; liens entre individus et entre groupes sociaux; eschatologie, etc. Elles permettent, dans le cadre d'un rituel aux phases bien définies, l'expression et la canalisation de la douleur des proches. Elles consacrent la séparation réciproque du défunt et de la communauté et permettent un certain nombre de réajustements familiaux, économiques, politiques. Elles sont le lieu privilégié de l'expression d'une solidarité érigée en règle. Pour le défunt, les funérailles constituent une condition à la réalisation de l'ultime phase d'un cycle qui va de la naissance à l'ancestralité. Leur bon déroulement est une condition sine qua non pour s'assurer la bienveillance des ancêtres, et par là même une condition à la survie du groupe.

Présente dès le décès et tout au long de la cérémonie, la musique s'adresse tant aux vivants qu'au(x) défunt(s). Elle est à la fois hommage au mort (louanges des cantateurs) et invitation à l'adresse des ancêtres (répertoires pentatoniques joués par l'ensemble lóbrí). Les ensembles musicaux ont un rôle signalétique, mais surtout ils servent de médium et de support à l'expression de la douleur contribuant par là même à la canaliser.

\section{Bibliographie}

DIETERLEN, G. (1950). Essai sur la religion bambara, Paris, Presses universitaires de France.

GBÃANغ̀ DABIRE, C. (1983). Nisaal. L'homme comme relation, tome 1 , thèse présentée à l'École des gradués de l'Université Laval pour l'obtention du grade de Philosophice Doctor (Ph.D.) par Constantin Gbãanè Dabire, maître d'enseignement, section philosophie de l'Université de Strasbourg.

GOODY, J. (1962). Death, Property and the Ancestors. A Study of the Mortuary Customs of the LoDagaa of West Africa, Stanford, Stanford University Press.

SOMÉ, D. et J.-M. BEKUONE (dir.) (1976). Jalons pour une ethnomusicologie dagara. Enquêtes livrées par une équipe de chercheurs, sous commission de musique sacrée, Diébougou, Diocèse de Diébougou.

THOMAS, L.-V. (1982). La mort africaine. Idéologie funéraire en Afrique noire, Paris, Payot.

\section{Notes}

1. Remerciements à Susanne Fürniss, chercheur au CNRS (Unité Mixte de Recherche 8099, LMS) pour son exigence toujours constructive, pour sa patience et sa compréhension lors de la direction de mes recherches, à Michaël Houseman, directeur d'études à l'EPHE $\mathrm{V}^{\mathrm{e}}$ section, pour ses précieux conseils, ainsi qu'à Sylvie Paz, Élise Heinisch et Jeanne Miramon-Bonhoure pour leurs relectures et conseils. Ce sont mes photos qui accompagnent le texte.

2. En dàgàrà, le terme générique pour «xylophone» est jìl, parfois orthographié gyil.

3. Une échelle pentatonique hémitonique est une échelle de cinq sons comportant un ou plusieurs demi-tons.

4. Échelle de cinq sons sans demi-ton.

5. Louanges, paroles de consolation, lamentations. On peut entendre un làngnìn sur le CD qui accompagne ce numéro de Frontières.

6. De làng: ensemble, et kónné que les Dàgàrà traduisent par «cantater», désignant par là l'activité même des làng-kóndbè.

7. L'origine de l'utilisation de ce terme par les Dàgàrà ne m'est pas connue.

8. Il est important de noter ici que la question de savoir si l'on a affaire dans le cas des cantateurs à de la musique ou non n'est très probablement pas une question que se posent les Dàgàrà. Je leur ai en l'occurrence moi-même posé la question en français. Notons qu'il n'existe pas de terme Dàgàrà pour "musique » et que «chant» se dit yìelù.

9. Le terme «responsorial» renvoie initialement au chant liturgique chrétien primitif alterné entre l'assemblée des fidèles et un ou plusieurs chantres. De nos jours, on emploie le plus souvent le terme "responsorial» lorsque le chant est alterné entre un ou plusieurs solistes et un chœur, et «antiphonal » lorsque le chant se répartit entre deux chœurs.

10. En comptant à partir des aigus.

11. Afin d'alléger la lecture, je parlerai par la suite de «3 dìgàar» (ou 4) et de "un bèlàngnèn ». Notons qu'il y a toujours une pause entre les pièces du répertoire dغ̀gàar et que celles du répertoire bèlàngnèn sont toujours enchâ̂nées les unes aux autres. J'ignore quelle est la raison de ce fait, s'il y en a une.

12. Je reprends ce terme à Constantin Gbãanè Dabire qui parle de "scènes de commémorations » (Gbãanè Dabire, 1983, p. 275).

13. Constantin Gbãanè Dabire précise qu'il est de coutume «que certains enterrements se fassent de nuit (pour un vieillard par exemple)» (Gbãanદ̀ Dabire, 1983, p. 282).

14. Les sources divergent sur ce point. 